\title{
Is the $\mathrm{B} / \mathrm{C}$ data slope in AMS-02 data actually telling us something about the diffusion coefficient slope?
}

\author{
P.-I. Batista, M. Vecchi*, D. Maurin, L. Derome, M. Boudaud, Y. Génolini, J. Lavalle, \\ V. Poulin, P. Salati, P. D. Serpico \\ University of Groningen, The Netherlands, and Instituto de Física de São Carlos, Universidade \\ de São Paulo, CP 369, 13560-970, São Carlos, SP, Brazil \\ E-mail: m.vecchi@rug.nl
}

\begin{abstract}
The measurement of the boron to carbon flux ratio provides relevant insights on the propagation of cosmic rays in the Galaxy. There is a common misconception about the fact that high-energy B/C data from AMS-02 directly provides the slope of the diffusion coefficient, implying that additional effects at play (convection, reacceleration, and destruction) can be neglected.

Using the USINE code and taking into account all the relevant processes, we show that this assumption is not supported: the $\mathrm{B} / \mathrm{C}$ data slope is not constant, its rigidity dependence being shaped in particular by inelastic interactions up to $\mathrm{TeV}$ energies. Heavier species are expected to be more impacted than lighter ones, and this should reflect in AMS-02 data, for which a softening of the slope with $\mathrm{Z}$ is predicted.
\end{abstract}

36th International Cosmic Ray Conference -ICRC2019-

July 24th - August 1st, 2019

Madison, WI, U.S.A.

\footnotetext{
*Speaker.
} 


\section{Introduction}

The current generation of space-based cosmic-ray (CR) experiments has been providing for the first time percent level precision measurements [1]. These new observations have revealed subtle and unexpected spectral features [2] that require to reexamining or at least improving the theoretical framework used to describe the CR origin and propagation. The most promising observable to study cosmic-ray transport consists in the study of secondary species, or of the flux ratio of a secondary species, like Boron, to a (mostly) primary one, like Carbon. Recent measurements of the ratio between the Boron and the Carbon flux (B/C in the following) were provided by the PAMELA [3] as well as by the AMS-02 experiment [4]. Based on the B/C measurement published by AMS-02, the first evidence for a break in the diffusion coefficient [6] was inferred: this break is due to the diffusion effects rather than to source effects.

This work is organized as follows: in section 2 we give a short description of the general framework used for our study, while in section 3 we discuss the method and the results for the $\mathrm{B} / \mathrm{C}$, both in a (simplified) pure diffusion scenario, and in the full transport model. In section 4 we extend our study to all $\mathrm{CR}$ nuclei species, from $\mathrm{H}$ to $\mathrm{Fe}$, while in 5 we present our conclusions.

\section{Transport models}

In this section we introduce the transport equation that we solve semi-analytically with the USINE code [7]. The general formalism that describes the CR transport in the galaxy [8] relies on the following diffusion-advection equation for a CR species of index $\alpha$, extensively discussed in [9]. In the steady-state approximation and in energy space (rather than rigidity or momentum space) the transport equation reads:

$$
\begin{aligned}
& -\vec{\nabla}_{\mathbf{x}}\left\{K(E) \vec{\nabla}_{\mathbf{x}} \psi_{\alpha}-\vec{V}_{\mathrm{c}} \psi_{\alpha}\right\}+E\left\{b_{\mathrm{tot}}(E) \psi_{\alpha}-\beta^{2} K_{p p} \psi_{\alpha} E\right\}+\sigma_{\alpha} v_{\alpha} n_{\mathrm{ism}} \psi_{\alpha}+\Gamma_{\alpha} \psi_{\alpha} \\
& =q_{\alpha}+\sum_{\beta}\left\{\sigma_{\beta \rightarrow \alpha} v_{\beta} n_{\text {ism }}+\Gamma_{\beta \rightarrow \alpha}\right\} \psi_{\beta} .
\end{aligned}
$$

This equation describes the spatial and energy evolution of the differential interstellar CR density per unit energy $\psi_{\alpha} \equiv d n_{\alpha} / d E$, assuming a net primary injection rate of $q_{\alpha}$, and a secondary injection rate arising from inelastic processes converting heavier species of index $\beta$ into $\alpha$ species (with a production rate $\sigma_{\beta \rightarrow \alpha} v_{\beta} n_{\text {ism }}$ on the ISM density $n_{\text {ism }}$, or a decay rate $\Gamma_{\beta \rightarrow \alpha}$ ). This source term is balanced by several other terms, among which the decay rate $\Gamma_{\alpha}$ (if relevant). The central piece of the propagation equation is the spatial diffusion coefficient $K$, that we discuss in more detail in the following. The other processes are mostly relevant at low rigidity, but may still affect the determination of higher-energy parameters: convection is featured by a velocity $\vec{V}_{\mathrm{c}}$, diffusive reacceleration is parameterized by the energy-dependent coefficient $K_{p p}$, and the inelastic destruction rate is given by $\sigma_{\alpha} v_{\alpha} n_{\text {ism }} \psi_{\alpha}$, with the $\sigma$ 's being energy-dependent nuclear cross sections; energy losses are characterized by the rate $b_{\text {tot }} \equiv d E / d t$, which includes ionization and Coulomb processes as well as adiabatic losses induced by convection and reacceleration. Following the work presented in [9], we assume a 1D propagation model, where the magnetic halo confining the CRs is an infinite slab in the radial direction and of half-height $L$, whose value is fixed to $10 \mathrm{kpc}$, and was found to have a negligible impact on the results. In this frame, the vertical coordinate $z$ is 


\begin{tabular}{|c||c|c|c|}
\hline \hline Free parameters / Models & BIG & SLIM & QUAINT \\
\hline \hline$K_{10}$ & $\checkmark$ & $\checkmark$ & $\checkmark$ \\
$\delta$ & $\checkmark$ & $\checkmark$ & $\checkmark$ \\
\hline$\eta$ & 1 or $\checkmark$ & 1 & $\checkmark$ \\
$\delta_{1}$ & $\checkmark$ & $\checkmark$ & N/A \\
$s_{1}$ & 0.05 & 0.05 & N/A \\
$R_{1}$ & $\checkmark$ & $\checkmark$ & N/A \\
\hline$V_{\mathrm{A}}$ & $\checkmark$ & N/A & $\checkmark$ \\
$V_{\mathrm{c}}$ & $\checkmark$ & N/A & $\checkmark$ \\
\hline \hline
\end{tabular}

Table 1: Free parameters of the three benchmark models BIG, SLIM, and QUAINT, presented in [9]. The first block of parameters is associated with the diffusion coefficient in the intermediate regime, and is common to all models. The second block is related to a potential low-rigidity break in the diffusion coefficient, or to purely non-relativistic effects. The last block is related to reacceleration and convection.

the only relevant spatial coordinate, and the galactic disk, where the sources of CRs as well as the interstellar medium gas lie has an effective half-height $h=100 p c$; the observer is located at $\mathrm{z}=$ 0 . Energy losses are also considered to be localized in the disk, based on the approach initially presented in [10].

The diffusion coefficient is a key physical ingredient to describe CR transport. It describes the scattering of CRs off magnetic turbulence. It depends on the rigidity, and we assume that it can be described as a scalar function, homogeneous and isotropic all over the magnetic slab. In this work we use the general form presented in [9], that features a break in both the low and high rigidity range:

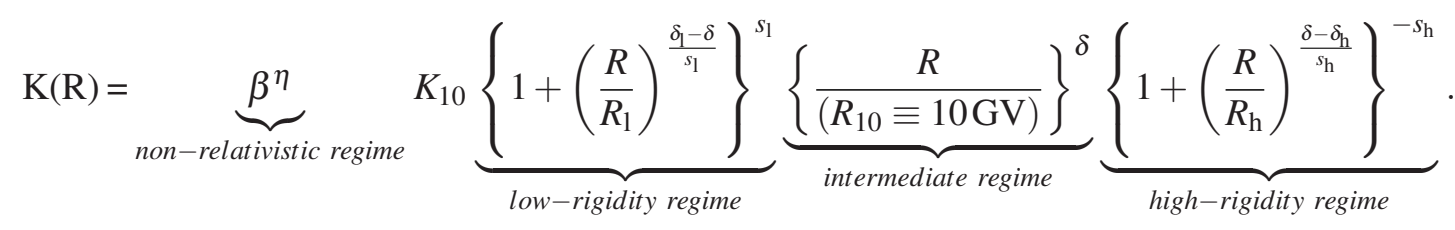

A new propagation model (dubbed BIG in the following) was first proposed in [9]. It includes a diffusion coefficient with a double break, as well as convection and reacceleration. The corresponding propagation parameters are displayed in table 1, together with the propagation parameters for two limiting cases (called SLIM and QUAINT). The SLIM model is a subpart of BIG, in which the low energy effects, convection and reacceleration, are discarded. The QUAINT model is also a subpart of the BIG model, but it describes the low energy data by means of a strong reacceleration, as well as a non-trivial, negative, value of the parameter $\eta$. For the nuclear production and spallation cross section we use as reference the set of tables from the Galprop package, following the approach described in [11].

\section{Is the $B / C$ data slope the same as the diffusion coefficient slope?}

In the simplest transport model, not anymore supported by the high precision AMS-02 data [4] [5], the propagation is a purely diffusive process, described by a feature-less diffusion coefficient. The 


\begin{tabular}{|c||c|c|}
\hline \hline Free parameters / Models & BIG & BIG Pure diff \\
\hline \hline$K_{10}\left[k p c^{2}\right]$ & 0.30 & 0.30 \\
$\delta$ & 0.48 & 0.48 \\
\hline$\eta$ & 1 or & 1 \\
$\delta_{1}$ & -0.69 & -0.69 \\
$s_{1}$ & 0.05 & 0.05 \\
$R_{1}[\mathrm{GV}]$ & 247 & 247 \\
\hline$V_{\mathrm{A}}[\mathrm{km} / \mathrm{s}]$ & 0 & N/A \\
$V_{\mathrm{c}}[\mathrm{km} / \mathrm{s}]$ & 67 & N/A \\
$\Delta_{\mathrm{h}}$ & 0.18 & 0.18 \\
\hline \hline
\end{tabular}

Table 2: Free parameters of the benchmark model BIG, together with the parameter values used in our study. The first block of parameters is associated with the diffusion coefficient in the intermediate regime, and is common to all models. The second block is related to a potential low-rigidity break in the diffusion coefficient, or to purely non-relativistic effects. The last block is related to reacceleration and convection.

primary CR fluxes can be described as $\psi_{P} \propto R^{-\alpha-\delta}, \alpha$ being the source spectral index and $\delta$ the diffusion coefficient spectral index. The fluxes of secondary species are expected to be shaped by diffusion, being softer by a factor $\delta$, i.e. $\psi_{S} \propto R^{-\alpha-2 \delta}$. The secondary-to-primary flux ratios, as the $\mathrm{B} / \mathrm{C}$, are thus expected to provide direct insights on the diffusion coefficient spectral index, being $\frac{\psi_{S}}{\psi_{P}} \propto R^{-\delta}$. In view of the recently reported evidence for a spectral break of diffusive origin, above the break the fluxes become harder by a quantity $\Delta \delta$.

The measured CR fluxes and flux ratios can be described as a power law in rigidity, i.e. $\phi \propto R^{-\gamma_{O b s}}$, the spectral index $\gamma_{O b s}$ being defined as:

$$
\gamma_{O b s}=d[\log \phi] / d[\log R]
$$

In this section $\phi$ indicates the $\mathrm{B} / \mathrm{C}$, since our goal is to compare this quantity to the diffusion coefficient spectral index $\delta$, that is fixed once the propagation model is selected, in order to assess whether or not these two quantities are equal. We define the following quantity:

$$
\Delta_{\text {slope }}=\gamma_{O b s}-\gamma_{T h}
$$

In the simplest pure diffusion transport model, we expect $\gamma_{T h}=\delta$ below the break, leading to $\Delta_{\text {slope }}=0$. Above the break $\Delta_{\text {slope }}=\Delta_{h}$ is expected. Figure 1 shows the USINE result for the B/C (top plot), together with the $\Delta_{\text {slope }}$ behaviour (bottom plot), as a function of rigidity. The $\mathrm{B} / \mathrm{C}$ prediction is obtained with the parameters described in right column of table 2 for the pure diffusion model. We can see that our result is in contrast with the expected behaviour for $\Delta_{\text {slope }}$, especially below the break, as a consequence of the production of secondary nuclei, while it reaches the expected $\Delta_{\text {slope }}=\Delta \delta$ above the break, around $1 \mathrm{TV}$. This simplified scenario already allows us to point out the fact that the $\mathrm{B} / \mathrm{C}$ and the diffusion coefficient spectral index are not interchangeable quantities. Figure 2 is obtained in the frame of the full transport equation. It shows the AMS-02 data together with $\mathrm{B} / \mathrm{C}$ predictions for the three benchmark models, in which convection, reacceleration, and destruction are properly taken into account. The bottom panel shows the $\Delta_{\text {slope }}$ for 


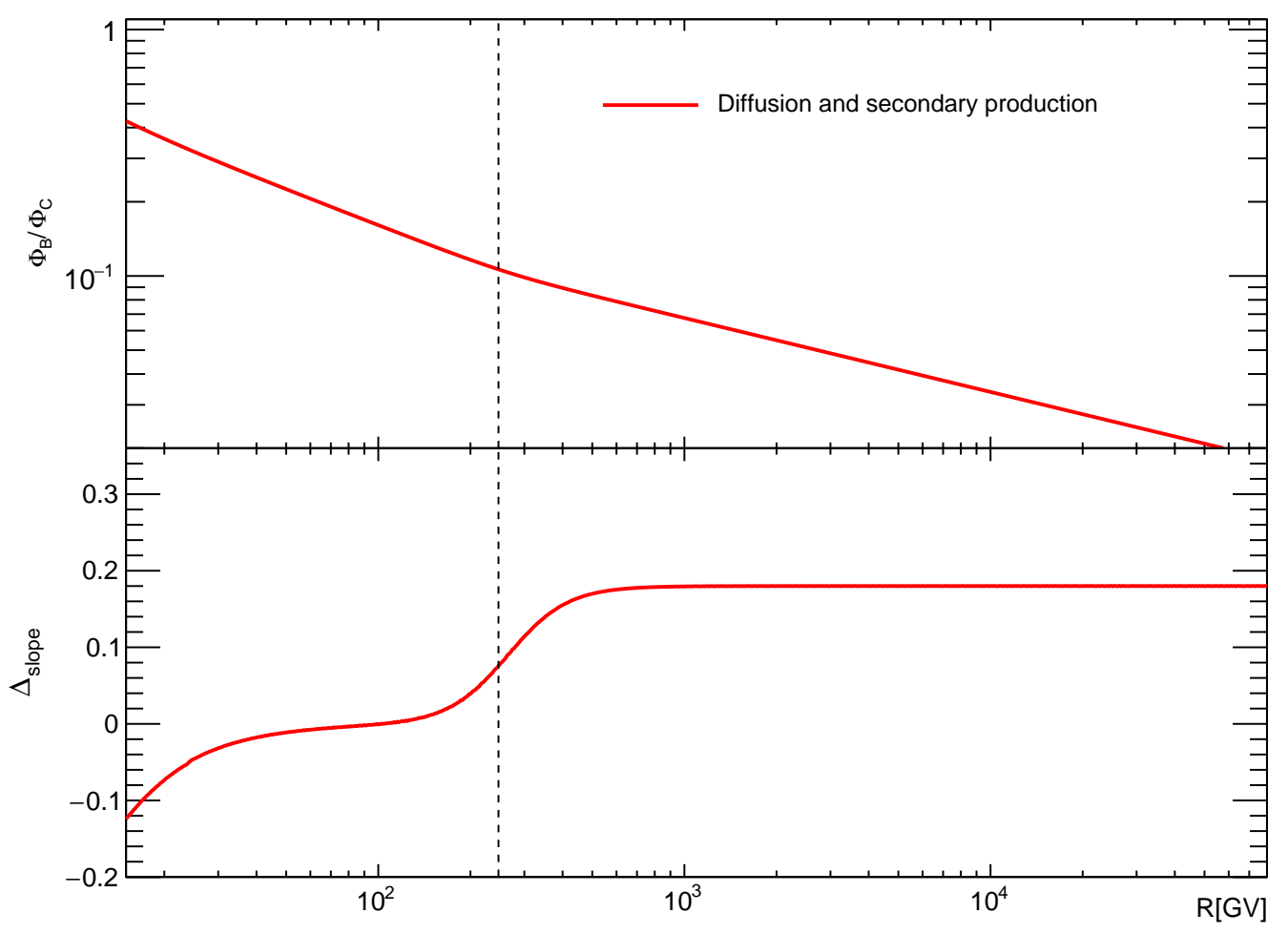

Figure 1: Pure diffusion propagation model. Top panel: The B/C flux ratio prediction as a function of rigidity. Bottom panel: $\Delta_{\text {slope }}$ as a function of rigidity. The position of the vertical dashed line indicates the high rigidity break in the diffusion coefficient.

the three benchmark models. This confirms the findings obtained already with the purely diffusive scenario, and shows that the slope of the $\mathrm{B} / \mathrm{C}$ should be disregarded as a direct indicator of the diffusion coefficient, because additional physical processes, in particular inelastic interactions, play a major role up the TV region.

\section{Flux slope behavior for different species}

In this section we investigate whether our findings also apply to other CR species. For all nuclei the injection is assumed to follow a universal power law of the rigidity, with $\alpha$ the common source spectral index. The results obtained for the pure diffusion scenario are shown in figure 3 as a function of the atomic number $\mathrm{Z}$. The green dashed line indicates the expected behavior for primary species, while the brown dotted line indicates the expected (softer) behavior for secondary species. The observed behaviour is due to the fact that only $\mathrm{H}, \mathrm{Si}$ and $\mathrm{Fe}$ are close to be fully primary species, while all other species including $\mathrm{He}, \mathrm{C}$ and $\mathrm{O}$, have a non negligible secondary component, coming from inelastic interactions of heavier species. Secondary components are expected to be softer than primaries, such that the spectral indices of all species are shaped by these phenomena. Moreover, the secondary production can take place in one or multiple steps, shaping the spectral index even strongly. The higher the fractional contribution of multi-step secondary production, the higher the deviation with respect to the expected behavior. Figure 4 shows the relative contributions 


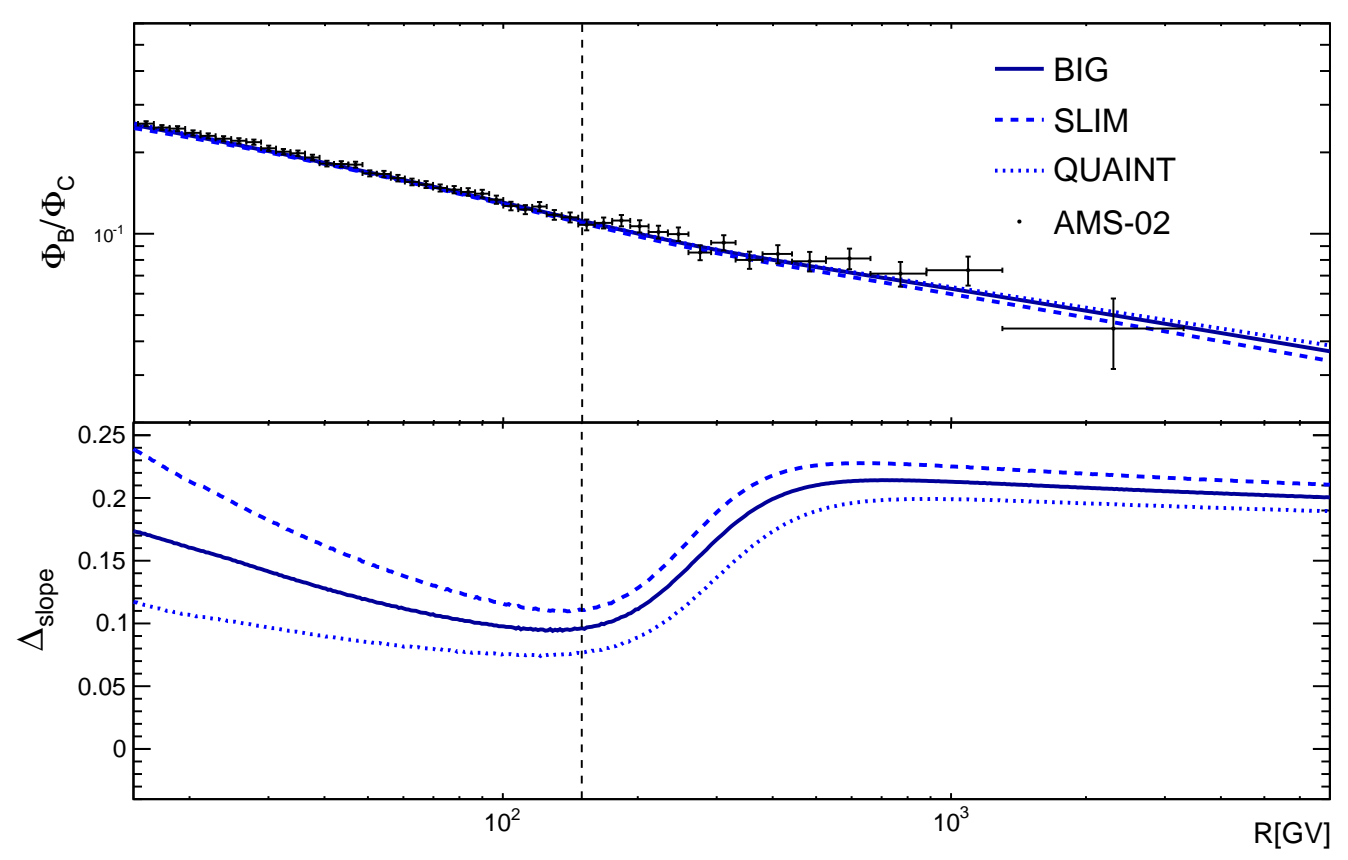

Figure 2: Full transport equation. Top panel: The B/C flux ratio prediction as a function of rigidity, for the three benchmark models, together with the AMS-02 data [5]. Bottom panel: $\Delta_{\text {slope }}$ as a function of rigidity, for the three benchmark models. The position of the vertical dashed line indicates $200 \mathrm{GV}$.

per production process for elemental fluxes (from $\mathrm{H}$ to $\mathrm{Fe}$ ). The species with the highest primary content are $\mathrm{H}, \mathrm{O}, \mathrm{Si}$, and $\mathrm{Fe}$ (black), while $\mathrm{Li}, \mathrm{Be}, \mathrm{B}, \mathrm{F}$, and $\mathrm{Cl}$ to $\mathrm{V}$ have the highest secondary component from both single (red) and multi-step production (blue and green). When all transport effects are accounted for, we find that inelastic collisions play the most relevant effect, further reducing the differences in the CR fluxes slopes. Figure 5 shows the behavior of the observed flux slope (at $50 \mathrm{GV}$ ) as a function of the atomic number Z, for the BIG model, together with AMS-02 results [5] (green squares). The difference with AMS-02 points might be related to the fact that AMS-02 points are from a fit to data above $50 \mathrm{GV}$ whereas our calculation is the local slope, so they are not exactly the same quantity. Proton and iron are not expected to have the same slope. Fe is expected to be harder, by $\sim 0.2$, because the inelastic cross-section is larger [12]. Furthermore, sub-Fe elements are expected to be harder than $\mathrm{Li}, \mathrm{Be}$ and $\mathrm{B}$ by roughly the same amount.

\section{Conclusions}

Assuming a 1D propagation model, we computed the B/C with the USINE code, assuming the BIG, SLIM and QUAINT propagation models. We found that the B/C spectral index should not be interpreted as the diffusion coefficient spectral index, since additional physical processes, especially inelastic collisions with the ISM, play an important role and cannot be neglected. Moreover, we computed the spectral index of the fluxes as a function of the atomic number for the BIG model, and found that the flux spectral index differs significantly from the expected values, for both primaries and secondaries. While in the pure diffusion scenario $\gamma_{O b s}$ is softer than the predictions, 


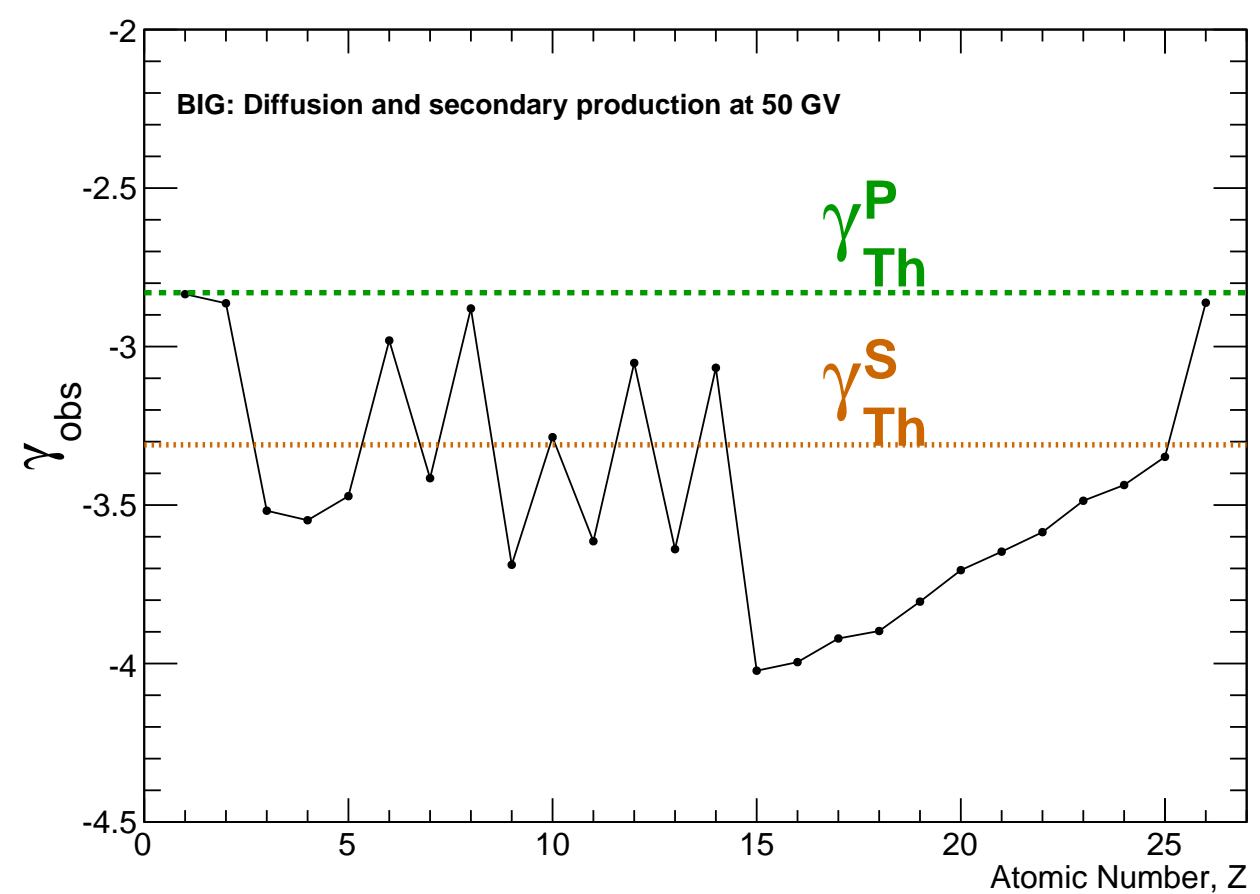

Figure 3: Behavior of the observed flux slope, computed at $50 \mathrm{GV}$, as a function of the atomic number Z, in the frame of the simplified pure diffusion model. The green dashed line indicates the expected behavior for primary species, while the brown dotted line indicates the expected behavior for secondary species.

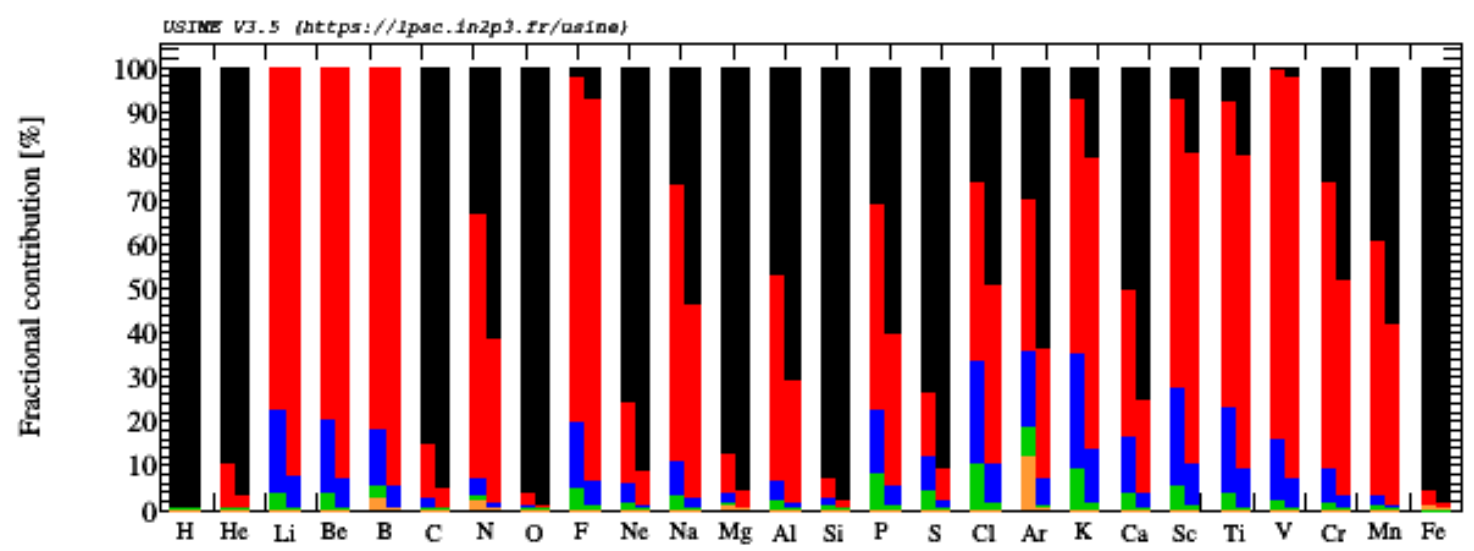

Figure 4: Relative contributions per production process for elemental fluxes (isotopes not shown) at 50 and 2000 TV (isotopes not shown): primary (black), secondary (1, 2, and $>2$ steps in red, blue, and green), radioactive (orange).

adding convection, reacceleration and inelastic collisions results in a flux hardening, compatible with the spectral indices estimated by the AMS- 02 collaboration. The forthcoming publications of heavy nuclei $(Z>9)$ measured by AMS-02 will be very helpful to further probe the model. 


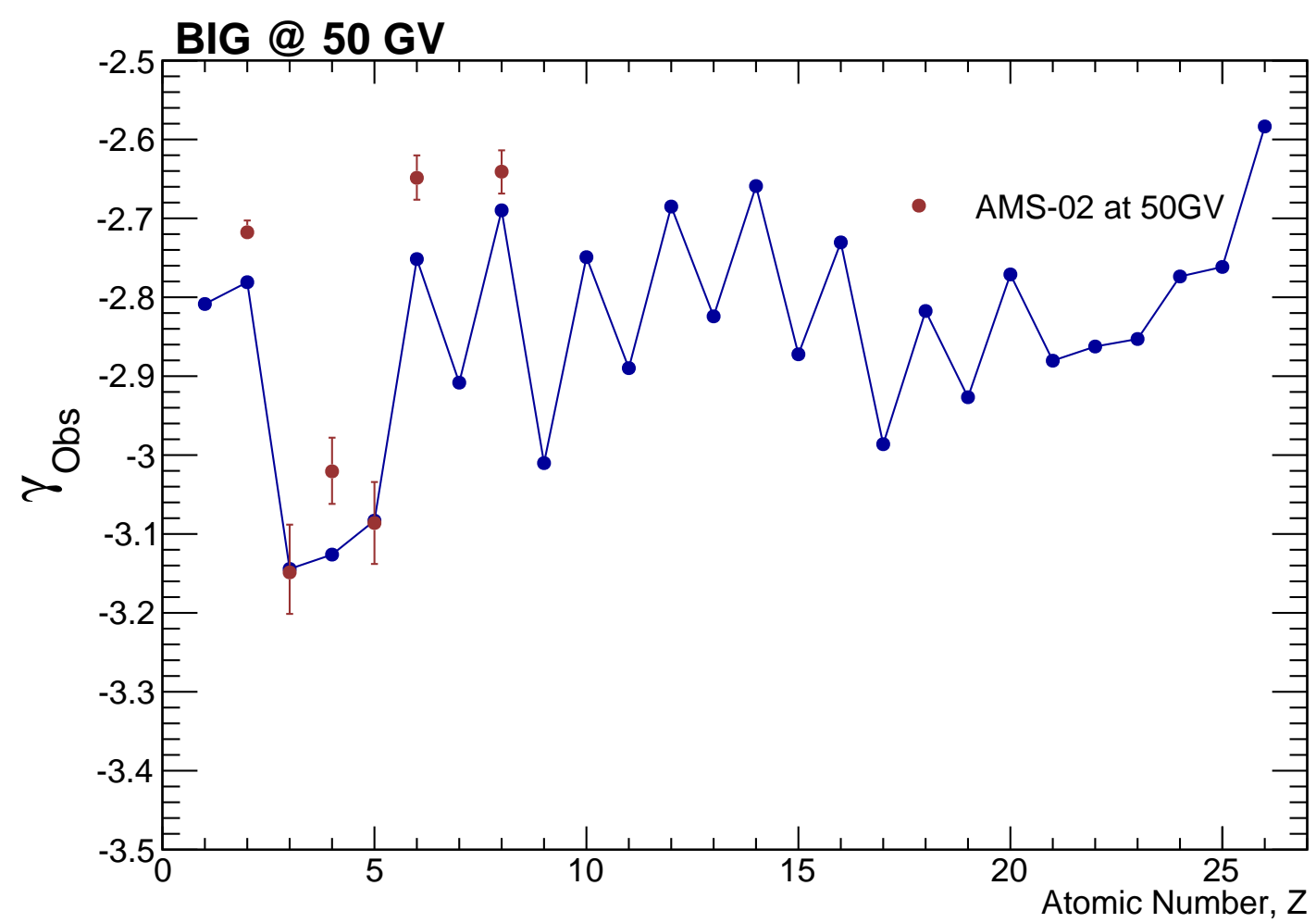

Figure 5: Behavior of the observed CR flux slope, computed at $50 \mathrm{GV}$, as a function of the atomic number $\mathrm{Z}$, in the frame of the BIG model. The red dots show the AMS-02 results [5].

\section{References}

[1] P. S. Marrocchesi, arXiv:1704.00304 [astro-ph.HE].

[2] P. D. Serpico, PoS ICRC 2015 (2016) 009

[3] O. Adriani et al., Astrophys. J. 791 (2014) no.2, 93

[4] M. Aguilar et al. [AMS Collaboration], Phys. Rev. Lett. 117 (2016) no.23, 231102.

[5] M. Aguilar et al. [AMS Collaboration], Phys. Rev. Lett. 120 (2018) no.2, 021101.

[6] Y. Génolini et al., Phys. Rev. Lett. 119 (2017) no.24, 241101

[7] D. Maurin, arXiv:1807.02968

[8] E. Amato and P. Blasi, Adv. Space Res. 62 (2018) 2731

[9] Y. Génolini et al., Phys. Rev. D 99 (2019) no.12, 123028

[10] M. Boudaud et al., Astron. Astrophys. 605 (2017) A17

[11] L. Derome et al., arXiv:1904.08210

[12] Y. Génolini, D. Maurin, I. V. Moskalenko and M. Unger, Phys. Rev. C 98 (2018) no.3, 034611 